\title{
LENS-SHAPED REGIONS FOR STRONG STIELTJES MOMENT PROBLEMS
}

\section{Olav Njåstad}

Dedicated to Professor Richard Askey on the occasion of his 65th birthday.

\begin{abstract}
It is shown that the values of the Stieltjes transform of solutions of the strong (or two-sided) Stieltjes moment problem cover lens-shaped regions determined by two related strong Hamburger moment problems. Conditions for determinacy of the strong Stieltjes moment problem are given.
\end{abstract}

\section{Introduction}

The classical Stieltjes moment problem can be described as follows: let a sequence $\left\{c_{n}\right\}_{n=0}^{\infty}$ of real numbers be given. Find positive measures $\mu$ on the non-negative real axis such that

$$
c_{n}=\int_{0}^{\infty} t^{n} d \mu(t) \text { for } n=0,1,2, \ldots
$$

Similarly, the classical Hamburger moment problem consists of finding positive measures $\mu$ on the whole real axis such that

$$
c_{n}=\int_{-\infty}^{\infty} t^{n} d \mu(t) \text { for } n=0,1,2, \ldots
$$

These problems were treated first by Stieltjes [39] in 1894 and then by Hamburger [15] in 1920-1921. These initial works were followed by an extensive development of a theory of moment problems where the connection with the theory of orthogonal polynomials plays a central role. See [1-5, 8-10, 12-13, 22, 25-26, 33-35, 40-42].

The strong Stieltjes moment problem (SSMP) and strong Hamburger moment problem (SHMP) are defined analogously for doubly infinite sequences:

Let a double sequence $\left\{c_{n}\right\}_{n=-\infty}^{\infty}$ of real numbers be given. Find positive measures $\mu$ on the non-negative real axis or on the whole real axis such that

$$
c_{n}=\int_{0}^{\infty} t^{n} d \mu(t) \quad \text { for } \quad n=0, \pm 1, \pm 2, \ldots
$$

or

$$
c_{n}=\int_{-\infty}^{\infty} t^{n} d \mu(t) \quad \text { for } \quad n=0, \pm 1, \pm 2, \ldots
$$

respectively.

Received March 16, 1998, revised September 9, 1998.

1991 Mathematics Subject Classification: 30E05, 42C05, 44A60.

Key words and phrases: strong moment problems, orthogonal Laurent polynomials. 
These problems were introduced around 1980 by Jones et al. [21] for the Stieltjes case, and by Jones and Thron [19-20] for the Hamburger case. A theory of these problems and their connection with a theory of orthogonal Laurent polynomials has been developed. This theory is, to a large extent, parallel to the classical theory, but there also appear phenomena that do not seem to have obvious classical analogues. We also mention that just as the classical theory of the Stieltjes moment problem is closely connected with the theory of Padé approximation, so the theory of the strong Stieltjes moment problem is closely connected with the theory of two-point Padé approximation; see [16] for further references. This is a special case of the very general theory of multipoint Padé approximation, originally studied by Gonchar and Lopez; see [14, 23-24] for further references, and also [7].

As in the classical situation, a moment problem is called determinate if it has exactly one solution, indeterminate if it has more than one solution. The values at an arbitrary point $z$ outside the real axis of the Stieltjes transform of all solutions of an indeterminate classical Hamburger moment problem exactly cover a proper disk. See $[1,3,26,37-38]$. The values of the Stieltjes transform of all solutions of an indeterminate classical Stieltjes moment problem exactly cover a lens-shaped region. See $[12,41]$, where more general situations are considered. Here the Stieltjes transform $F(z, \mu)$ of a measure $\mu$ is defined by

$$
F(z, \mu)=\int_{-\infty}^{\infty} \frac{d \mu(t)}{t-z} .
$$

For the SHMP, an analog of the classical result has been proved (see [17, 29, 32]): the values at an arbitrary point outside the real axis of the Stieltjes transform of all solutions of an indeterminate SHMP exactly cover a proper disk. In this paper, we obtain a result on lens-shaped regions for indeterminate SSMPs analogous to the classical one and discuss criteria for determinacy of SSMPs. We make some use of properties of two interrelated disk systems associated with a SSMP, as discussed in $[27,31]$.

\section{Orthogonal Laurent polynomials}

An important tool in the study of strong moment problems is the theory of orthogonal Laurent polynomials. A Laurent polynomial is a finite linear combination of monomials $z^{n}, n=0, \pm 1, \pm 2, \ldots$. For any pair $(p, q)$ of integers with $p \leq q$, we define

$$
\Lambda_{p, q}=\operatorname{Span}\left\{z^{p}, \ldots, z^{q}\right\}
$$

where the span is taken over the complex numbers.

Thus the space $\Lambda$ of all Laurent polynomials is the union of all the spaces $\Lambda_{p, q}$.

Let a doubly infinite sequence $\left\{c_{n}\right\}_{n=-\infty}^{\infty}$ be given. For simplicity, we assume that the sequence is normalized such that $c_{0}=1$, but this is not essential. We define a linear functional $M$ on $\Lambda$ by its value on the basis elements $z^{n}$ :

$$
M\left[z^{n}\right]=c_{n}, \quad n=0, \pm 1, \pm 2, \ldots
$$

We shall, in the following, always assume that $M$ is positive on $(0, \infty)$ (i.e., $M[f]>$ 0 for all $f \in \Lambda$ which satisfy $f(t) \geq 0$ for $t>0, f(t) \not \equiv 0)$. The SSMP for the double sequence $\left\{c_{n}\right\}$ then is solvable. Thus, there exists at least one measure $\mu$ on $[0, \infty)$ 
such that

$$
M[f]=\int_{0}^{\infty} f(t) d \mu(t) \quad \text { for all } f \in \Lambda .
$$

An inner product on the space of real Laurent polynomials is defined by

$$
\langle P, Q\rangle=M[P(x) Q(x)] .
$$

By orthogonalization of the basis sequence

$$
\left\{1, z^{-1}, z, z^{-2}, z^{2}, \ldots\right\}
$$

with respect to this inner product, we obtain an orthonormal sequence $\left\{\varphi_{n}\right\}_{n=0}^{\infty}$ of Laurent polynomials. They satisfy (with $\perp$ denoting orthogonality)

$$
\begin{gathered}
\varphi_{2 m} \in \Lambda_{-m, m}, \quad \varphi_{2 m} \perp \Lambda_{-m, m-1}, \quad m=1,2, \ldots, \\
\varphi_{2 m+1} \in \Lambda_{-(m+1), m}, \quad \varphi_{2 m+1} \perp \Lambda_{-m, m}, \quad m=0,1,2, \ldots
\end{gathered}
$$

These functions may be written in the form

$$
\begin{aligned}
\varphi_{2 m}(z) & =\frac{u_{2 m}}{z^{m}}+\cdots+v_{2 m} z^{m}, \\
\varphi_{2 m+1}(z) & =\frac{v_{2 m+1}}{z^{m+1}}+\cdots+u_{2 m+1} z^{m} .
\end{aligned}
$$

The coefficients $v_{n}$ are called leading coefficients, and the coefficients $u_{n}$ are called the trailing coefficients. They satisfy

$$
v_{n}>0, \quad(-1)^{n} u_{n}>0
$$

The associated orthogonal Laurent polynomials $\psi_{n}$ are defined by

$$
\psi_{n}(z)=M\left[\frac{\varphi_{n}(t)-\varphi_{n}(z)}{t-z}\right], \quad n=0,1,2, \ldots
$$

(the argument considered as a function of $t$ ). The sequences $\left\{\varphi_{n}\right\},\left\{\psi_{n}\right\}$ satisfy the following three-term recurrence relation:

$$
\begin{gathered}
{\left[\begin{array}{l}
\psi_{n}(z) \\
\varphi_{n}(z)
\end{array}\right]=\left(g_{n}+h_{n} z^{(-1)^{n}}\right)\left[\begin{array}{l}
\psi_{n-1}(z) \\
\varphi_{n-1}(z)
\end{array}\right]+f_{n}\left[\begin{array}{l}
\psi_{n-2}(z) \\
\varphi_{n-2}(z)
\end{array}\right], \quad n=1,2, \ldots,} \\
\varphi_{-1}=0, \quad \varphi_{0}=1, \quad \psi_{-1}=\frac{1}{z}, \quad \psi_{0}=0 .
\end{gathered}
$$

The coefficients $f_{n}, g_{n}, h_{n}$ are given in terms of leading and trailing coefficients by

$$
\begin{aligned}
& f_{1}=u_{1}, \quad f_{n}=\frac{u_{n} v_{n-2}}{u_{n-1} v_{n-1}}, \quad n=2,3, \ldots, \\
& g_{1}=u_{1}, \quad g_{n}=\frac{u_{n}}{v_{n-1}}, \quad n=2,3, \ldots, \\
& h_{1}=v_{1}, \quad h_{n}=\frac{v_{n}}{u_{n-1}}, \quad n=2,3, \ldots
\end{aligned}
$$

The quasi-orthogonal Laurent polynomials $\varphi_{n}(z, \tau)$ of order $n$ are defined by

$$
\varphi_{n}(z, \tau)=\varphi_{n}(z)-\tau z^{(-1)^{n}} \varphi_{n-1}(z) .
$$

Here $\tau \varepsilon \hat{\mathbb{R}}=\mathbb{R} \cup\{\infty\}$. (For $\tau=\infty, \varphi_{n}(z, \tau)$ means $-\varphi_{n-1}(z)$. Similar conventions are used throughout.) These functions play a similar role in the theory of orthogonal Laurent polynomials as the quasi-orthogonal polynomials do in the classical theory of 
orthogonal polynomials. The associated quasi-orthogonal Laurent polynomials $\psi_{n}(z, \tau)$ of order $n$ are defined by

$$
\psi_{n}(z, \tau)=\psi_{n}(z)-\tau z^{(-1)^{n}} \psi_{n-1}(z) .
$$

The pseudo-orthogonal Laurent polynomials $\Phi_{n}(z, \tau)$ of order $n$ are defined by

$$
\Phi_{n}(z, \tau)=\varphi_{n}(z)-\tau \varphi_{n-1}(z) .
$$

These expressions are formally like the quasi-orthogonal polynomials in the classical theory, but they do not behave in the same way. The associated pseudo-orthogonal Laurent polynomials $\Psi_{n}(z, \tau)$ of order $n$ are defined by

$$
\Psi_{n}(z, \tau)=\psi_{n}(z)-\tau \psi_{n-1}(z) .
$$

For more information on fundamental properties of orthogonal Laurent polynomials and strong moment problems, we refer to $[11,18-19,27-30,32,36]$.

\section{Systems of circles}

For proofs and more detailed treatments of the topics discussed in this section, see $[17,27-32]$.

The quasi-approximants of the continued fraction determined by the recurrence formulas (2.12)-(2.13) are the quotients

$$
R_{n}(z, \tau)=\frac{\psi_{n}(z, \tau)}{\varphi_{n}(z, \tau)}
$$

The zeros $\xi_{k}^{(n)}(\tau)$ of $\varphi_{n}(z, \tau)$ are real and simple, and at least $n-1$ of them are positive. All the zeros are positive if and only if $\tau \in\left[h_{2 m}, \infty\right]$ when $n=2 m$ and $\tau \in\left[-\infty, h_{2 m+1}\right]$ when $n=2 m+1$. In particular, the values $\tau=0$ and $\tau=\infty$ belong to these $\tau$-intervals. (Note that $\tau=-\infty$ and $\tau=\infty$ represent the same point on $\hat{\mathbb{R}}$.) For the above values of $\tau$, quadrature formulas with nodes at $\xi_{k}^{(n)}(\tau)$ and positive weights define discrete measures $\mu_{\tau}^{(n)}$ with support in $[0, \infty)$ such that

$$
R_{n}(z, \tau)=-F\left(z, \mu_{\tau}^{(n)}\right) .
$$

For the remaining values of $\tau$, discrete (positive) measures $\mu_{\tau}^{(n)}$ satisfying (3.2) are still determined by quadrature formulas, but with support not contained in $[0, \infty)$.

For each $z$ outside $\hat{\mathbb{R}}$, the Möbius transformation

$$
\tau \longrightarrow w=w_{n}=-R_{n}(z, \tau)
$$

maps $\hat{\mathbb{R}}$ onto a circle in the open upper half-plane $\mathbb{U}$. We use the notation $\Delta_{n}(z)$ for the open disk bounded by this circle, $\partial \Delta_{n}(z)$ for the circle itself and $\overline{\Delta_{n}}(z)$ for the closed disk $\Delta_{n}(z) \cup \partial \Delta_{n}(z)$. We denote by $\Omega_{n}(z)$ the subarc of $\partial \Delta_{n}(z)$ corresponding to those $\tau$-values which determine measures on $[0, \infty)$.

The pseudo-approximants of the continued fraction determined by the formulas (2.12)-(2.13) are the quotients

$$
S_{n}(z, \tau)=\frac{\Psi_{n}(z, \tau)}{\Phi_{n}(z, \tau)} .
$$

The zeros $\zeta_{k}^{(n)}(\tau)$ of $\Phi_{n}(z, \tau)$ are real and simple, and at least $n-1$ of them are positive. All the zeros are positive if and only if $\tau \in\left[-\infty, g_{2 m}\right]$ when $n=2 m$ and $\tau \in\left[g_{2 m+1}, \infty\right]$ when $n=2 m+1$. The values $\tau=0$ and $\tau=\infty$ belong to these 
$\tau$-intervals. For the above values of $\tau$, quadrature formulas with nodes at $\zeta_{k}^{(n)}(\tau)$ and positive weights determine discrete measures $\sigma_{\tau}^{(n)}$ with support in $[0, \infty)$ such that

$$
S_{n}(z, \tau)=-F\left(z, \sigma_{\tau}^{(n)}\right) \text {. }
$$

For the remaining values of $\tau$, quadrature formulas determine general discrete measures with support not contained in $[0, \infty)$ such that $(3.5)$ holds. These measures are not positive measures.

For each $z$ outside $\hat{\mathbb{R}}$, the Möbius transformation

$$
\tau \longrightarrow \omega=\omega_{n}=-S_{n}(z, \tau)
$$

maps $\hat{\mathbb{R}}$ onto a circle in $\mathbb{U}$. We denote by $D_{n}(z)$ the open disk bounded by this circle, by $\partial D_{n}(z)$ the circle itself, and by $\overline{D_{n}}(z)$ the closed disk $D_{n}(z) \cup \partial D_{n}(z)$. We denote by $\Gamma_{n}(z)$ the subarc of $\partial D_{n}(z)$ corresponding to those $\tau$-values which determine positive measures $\sigma_{\tau}^{(n)}$ on $[0, \infty)$.

The following equalities hold:

$$
\begin{aligned}
& \Omega_{n}(z)=\partial \Delta_{n}(z) \cap \overline{D_{n-1}}(z), \\
& \Gamma_{n}(z)=\partial D_{n}(z) \cap \overline{\Delta_{n-1}}(z) .
\end{aligned}
$$

We note that $\mu_{0}^{(n)}=\sigma_{0}^{(n)}=\mu_{\infty}^{(n+1)}=\sigma_{\infty}^{(n+1)}$ and denote this measure by $\mu^{(n)}$. The arcs $\Omega_{n}(z)$ and $\Gamma_{n}(z)$ have a common end point at $F\left(z, \mu^{(n-1)}\right)$ and intersect at $F\left(z, \mu^{(n)}\right)$.

The sequences $\left\{\Delta_{n}(z)\right\}$ and $\left\{D_{n}(z)\right\}$ are nested, i.e.,

$$
\Delta_{n+1}(z) \subset \Delta_{n}(z), \quad D_{n+1}(z) \subset D_{n}(z) .
$$

We define the limiting disks $\overline{\Delta_{\infty}}(z)$ and $\overline{D_{\infty}}(z)$ by

$$
\overline{\Delta_{\infty}}(z)=\bigcap_{n=1}^{\infty} \overline{\Delta_{n}}(z), \quad \overline{D_{\infty}}(z)=\bigcap_{n=1}^{\infty} \overline{D_{n}}(z) .
$$

The limiting disk $\overline{\Delta_{\infty}}(z)$ consists of a single point for every $z$ (the limit point case) or a proper closed disk for every $z$ (the limit circle case). Also, each $\overline{D_{\infty}}(z)$ is either a single point or a proper closed disk. It can be shown that a similar invariance property holds for $\overline{D_{\infty}}(z)$ as for $\overline{\Delta_{\infty}}(z)$; we prove this in Section 5 .

The $\operatorname{arcs} \Omega_{n}(z)$ tend to a limiting subarc $\Omega_{\infty}(z)$ of $\partial \Delta_{\infty}(z)$, and the $\operatorname{arcs} \Gamma_{n}(z)$ tend to a limiting subarc $\Gamma_{\infty}(z)$ of $\partial D_{\infty}(z) .\left(\partial \Delta_{\infty}(z)\right.$ and $\partial D_{\infty}(z)$ denote the boundary of $\overline{\Delta_{\infty}}(z)$ and $\overline{D_{\infty}}(z)$, respectively.) The $\operatorname{arcs} \Omega_{\infty}(z)$ and $\Gamma_{\infty}(z)$ may (simultanously) reduce to a single point. For these arcs, we have

$$
\begin{aligned}
& \Omega_{\infty}(z)=\partial \Delta_{\infty}(z) \cap \overline{D_{\infty}}(z), \\
& \Gamma_{\infty}(z)=\partial D_{\infty}(z) \cap \overline{\Delta_{\infty}}(z) .
\end{aligned}
$$

The sequences $F\left(z, \mu^{(2 m)}\right)$ and $F\left(z, \mu^{(2 m+1)}\right)$ tend to the common end points of $\Omega_{\infty}(z)$ and $\Gamma_{\infty}(z)$.

For each point $w \in \partial \Delta_{\infty}(z)$, there is a sequence of measures $\left\{\mu_{\tau}^{(n)}\right\}$ tending to a solution $\mu$ of the SHMP such that $F(z, \mu)=w$. When $w \in \Omega_{\infty}(z)$, this $\mu$ is a solution of the SSMP. The set of solutions of the SHMP is convex; therefore, there is for each $w \in \overline{\Delta_{\infty}}(z)$ at least one solution $\mu$ of the problem with $F(z, \mu)=w$. It can be shown that $F(z, \mu) \in \overline{\Delta_{\infty}}(z)$ for every solution $\mu$; see [32]. Thus, the values of the Stieltjes transform at $z$ of all solutions of the SHMP exactly cover $\overline{\Delta_{\infty}}(z)$. 
For each point $\omega \in \Gamma_{\infty}(z)$, there is a sequence of measures $\left\{\sigma_{\tau}^{(n)}\right\}$ tending to a solution $\sigma$ of the SSMP such that $F(z, \sigma)=\omega$. In particular, the sequences $\left\{\mu^{(2 m)}\right\}$ and $\left\{\mu^{(2 m+1)}\right\}$ converge to solutions $\mu^{(0)}$ and $\mu^{(\infty)}$, with $F\left(z, \mu^{(0)}\right)$ and $F\left(z, \mu^{(\infty)}\right)$ as the common end points of $\Omega_{\infty}(z)$ and $\Gamma_{\infty}(z)$.

Let $\overline{L_{\infty}}(z)$ denote the lens-shaped region bounded by the $\operatorname{arcs} \Omega_{\infty}(z)$ and $\Gamma_{\infty}$, i.e.,

$$
\overline{L_{\infty}}(z)=\overline{\Delta_{\infty}}(z) \cap \overline{D_{\infty}}(z) .
$$

The set of solutions of the SSMP is convex; therefore, it follows from the foregoing that for every point $w$ in $\overline{L_{\infty}}(z)$, there is a solution $\mu$ of the SSMP such that $F(z, \mu)=$ $w$. Thus, the region $\overline{L_{\infty}}(z)$ is covered by values of the Stieltjes transform at $z$ of solutions of the SSMP. We shall prove in Section 5 that all such values exactly cover $\overline{L_{\infty}}(z)$.

Remark 1. The argument above shows that the values of the Stieltjes transform cover the convex hull of $\overline{L_{\infty}}(z)$. It follows from Theorem 6.1 that all the values of the transform are contained in $\overline{L_{\infty}}(z)$; hence, the region $\overline{L_{\infty}}(z)$ is convex.

\section{Shifted moment problems}

As before, we assume that the sequence $\left\{c_{n}\right\}_{n=-\infty}^{\infty}$ determines a solvable SSMP. We now define

$$
\gamma_{n}=c_{n+1}, \quad n=0, \pm 1, \pm 2, \ldots
$$

We see that if $\mu$ is a solution of the SSMP for $\left\{c_{n}\right\}$, then the measure given by

$$
d \nu(t)=t d \mu(t)
$$

is a solution of the SSMP for $\left\{\gamma_{n}\right\}$, and vice versa. Thus, the formula (4.2) describes a one-to-one correspondence between solutions of the SSMP for $\left\{c_{n}\right\}$ and for $\left\{\gamma_{n}\right\}$.

The linear functional $L$ on $\Lambda$ defined by $\left\{\gamma_{n}\right\}$ determines an inner product with an orthogonal sequence associated with the basis (2.5). (Each element then is determined up to a constant.) We shall express these orthogonal Laurent polynomials in terms of the Laurent polynomials $\left\{\varphi_{n}\right\}$. All measures which solve a given moment problem give rise to the same inner product, that determined by the functional. For notational convenience, we shall use measures in the orthogonality arguments. Thus, in the following, let $\mu$ be a solution of the SSMP associated with $\left\{c_{n}\right\}$, and let $\nu$ be given by (4.2).

We define

$$
\begin{aligned}
\pi_{2 m-1}(z) & =\frac{1}{z}\left[\varphi_{2 m}(z)-g_{2 m} \varphi_{2 m-1}(z)\right], \quad m=1,2, \ldots, \\
\pi_{2 m}(z) & =\varphi_{2 m}(z)+\frac{1}{g_{2 m}} \varphi_{2 m-1}(z), \quad m=1,2, \ldots
\end{aligned}
$$

(We set $\pi_{0}=\varphi_{0}$. The system $\left\{\pi_{n}\right\}$, in general, will not be normalized.)

Theorem 4.1. The sequence $\left\{\pi_{n}\right\}$ is an orthogonal sequence associated with the basis (2.5) with respect to $L$, i.e.,

$$
\begin{aligned}
\pi_{2 m-1} & \in \Lambda_{-m, m-1}, & \pi_{2 m-1} \perp \Lambda_{-(m-1), m-1}, \\
\pi_{2 m} & \in \Lambda_{-m, m}, & \pi_{2 m} \perp \Lambda_{-m, m-1}
\end{aligned}
$$

where the orthogonality $\perp$ is with respect to the functional $L$. 
Proof. It follows from the form of $\varphi_{n}(z)$ given in (2.8)-(2.9), together with (2.15), that $\pi_{2 m-1} \in \Lambda_{-m, m-1}$ and $\pi_{2 m} \in \Lambda_{-m, m}$.

Let $p \in\{-(m-1), \ldots, m-1\}$. We have

$$
\int_{0}^{\infty} x^{p} \pi_{2 m-1}(x) d \nu(x)=\int_{0}^{\infty} x^{p}\left[\varphi_{2 m}(x)-g_{2 m} \varphi_{2 m-1}(x)\right] d \mu(x) .
$$

It follows from (2.6)-(2.7) that both $\varphi_{2 m}$ and $\varphi_{2 m-1}$ are orthogonal to $x^{p}$ with respect to $\mu$. Consequently, $\pi_{2 m-1}$ is orthogonal to $\Lambda_{-(m-1), m-1}$ with respect to $\nu$.

Next, let $p \in\{-m, \ldots, m-1\}$. We have

$$
\int_{0}^{\infty} x^{p} \pi_{2 m}(x) d \nu(x)=\int_{0}^{\infty} x^{p+1}\left[\varphi_{2 m}(x)+\frac{1}{g_{2 m}} \varphi_{2 m-1}(x)\right] d \mu(x) .
$$

It follows from (2.6)-(2.7) that both $\varphi_{2 m}$ and $\varphi_{2 m-1}$ are orthogonal to $x^{p+1}$ with respect to $\mu$ for $p=-m, \ldots, m-2$; hence, $\pi_{2 m}$ is orthogonal to $\Lambda_{-m, m-2}$ with respect to $\nu$. We need to show that $\pi_{2 m}$ also is orthogonal to $x^{m-1}$.

We have

$$
\begin{aligned}
& \int_{0}^{\infty} x^{m-1} \pi_{2 m}(x) d \nu(x) \\
&=\int_{0}^{\infty} x^{m} \varphi_{2 m}(x) d \mu(x)+\frac{1}{g_{2 m}} \int_{0}^{\infty} x^{m} \varphi_{2 m-1}(x) d \mu(x) .
\end{aligned}
$$

We may write

$$
x^{m}=A_{m} \varphi_{2 m}(x)+B_{m} \varphi_{2 m-1}(x)+f(x)
$$

with $f \in \Lambda_{-(m-1), m-1}$. By comparing coefficients of $x^{m}$ and $x^{-m}$ in (4.10), we find that

$$
A_{m}=\frac{1}{v_{2 m}}, B_{m}=-\frac{u_{2 m}}{v_{2 m-1} v_{2 m}} .
$$

Consequently, by orthogonality

$$
\int_{0}^{\infty} x^{m} \varphi_{2 m}(x) d \mu(x)=\frac{1}{v_{2 m}} \int_{0}^{\infty}\left(\varphi_{2 m}(x)\right)^{2} d \mu(x)=\frac{1}{v_{2 m}}
$$

and

$$
\int_{0}^{\infty} x^{m} \varphi_{2 m-1}(x) d \mu(x)=-\frac{g_{2 m}}{v_{2 m}} \int_{0}^{\infty}\left(\varphi_{2 m-1}(x)\right)^{2} d \mu(x)=-\frac{g_{2 m}}{v_{2 m}} .
$$

By substituting from (4.12)-(4.13) into (4.9), we find that $\pi_{2 m}$ is orthogonal to $x^{m-1}$ with respect to $\nu$.

We denote by $\rho_{n}$ the Laurent polynomials associated with the orthogonal Laurent polynomials $\pi_{n}$ with respect to the functional $L$, or to the measure $\nu$, i.e.,

$$
\rho_{n}(z)=\int_{0}^{\infty} \frac{\pi_{n}(t)-\pi_{n}(z)}{t-z} d \nu(t)=\int_{0}^{\infty} \frac{\pi_{n}(t)-\pi_{n}(z)}{t-z} t d \mu(t) .
$$

The auxiliary functions $\omega_{n}$ are introduced as

$$
\begin{aligned}
\omega_{2 m-1}(z) & =\frac{1}{z}\left[\psi_{2 m}(z)-g_{2 m} \psi_{2 m-1}(z)\right], \\
\omega_{2 m}(z) & =\psi_{2 m}(z)+\frac{1}{g_{2 m}} \psi_{2 m-1}(z) .
\end{aligned}
$$


(These are easily seen to be the Laurent polynomials associated to $\pi_{n}$ with respect to $\mu$.) We shall show how to express the functions $\rho_{n}$ in terms of $\pi_{n}$ and $\omega_{n}$.

Theorem 4.2. The associated orthogonal Laurent polynomials $\rho_{n}(z)$ can be written as

$$
\rho_{n}(z)=-\pi_{n}(z)+z \omega_{n}(z), \quad n=1,2, \ldots
$$

Proof. We may write

$$
\frac{\left[\varphi_{n}(t)-\varphi_{n}(z)\right] t}{t-z}=\varphi_{n}(t)-\varphi_{n}(z)+\frac{z\left[\varphi_{n}(t)-\varphi_{n}(z)\right]}{t-z}
$$

hence,

$$
\int_{0}^{\infty} \frac{\left[\varphi_{n}(t)-\varphi_{n}(z)\right]}{t-z} t d \mu(t)=-\varphi_{n}(z)+z \psi_{n}(z)
$$

since $c_{0}=1$ and $\int_{0}^{\infty} \varphi_{n}(t) d \mu(t)=0$. We also may write

$$
\frac{z \varphi_{n}(t)-t \varphi_{n}(z)}{z(t-z)}=-\frac{1}{z} \varphi_{n}(z)+\frac{\varphi_{n}(t)-\varphi_{n}(z)}{t-z}
$$

hence,

$$
\int_{0}^{\infty} \frac{t^{-1} \varphi_{n}(t)-z^{-1} \varphi_{n}(z)}{t-z} t d \mu(t)=-\frac{1}{z} \varphi_{n}(z)+\psi_{n}(z)
$$

It follows that

$$
\begin{aligned}
\rho_{2 m-1}(z)= & \int_{0}^{\infty} \frac{t^{-1} \varphi_{2 m}(t)-z^{-1} \varphi_{2 m}(z)}{t-z} t d \mu(t) \\
& -g_{2 m} \int_{0}^{\infty} \frac{t^{-1} \varphi_{2 m-1}(t)-z^{-1} \varphi_{2 m-1}(z)}{t-z} t d \mu(t) \\
= & -\frac{1}{z} \varphi_{2 m}(z)+\psi_{2 m}(z)-g_{2 m}\left[-\frac{1}{z} \varphi_{2 m-1}(z)+\psi_{2 m-1}(z)\right] \\
= & -\pi_{2 m-1}(z)+z \omega_{2 m-1}(z) .
\end{aligned}
$$

Similarly,

$$
\begin{aligned}
\rho_{2 m}(z)= & \int_{0}^{\infty} \frac{\varphi_{2 m}(t)-\varphi_{2 m}(z)}{t-z} t d \mu(t) \\
& \quad+\frac{1}{g_{2 m}} \int_{0}^{\infty} \frac{\varphi_{2 m-1}(t)-\varphi_{2 m-1}(z)}{t-z} t d \mu(t) \\
= & -\varphi_{2 m}(z)+z \psi_{2 m}(z)+\frac{1}{g_{2 m}}\left[-\varphi_{2 m-1}(z)+z \psi_{2 m-1}(z)\right] \\
= & -\pi_{2 m}(z)+z \omega_{2 m-1}(z)
\end{aligned}
$$

This completes the proof.

\section{Shifted system of circles}

We shall now discuss the disk systems $\overline{\Theta_{n}}(z)$ and $\overline{T_{n}}(z)$ determined by the quasiapproximants and pseudo-approximants associated with the sequence $\left\{\gamma_{n}\right\}$. We observe that the circles and disks will be the same if in the definitions (3.1), (3.3), (3.4), and (3.6), the orthonormal functions are replaced by any orthogonal functions and their associated functions, given in analogy with (2.11). Thus, we may (by a slight shift 
of notation) write the quasi-approximants $r_{n}(z, \tau)$ and pseudo-approximants $s_{n}(z, \tau)$ associated with $\left\{\gamma_{n}\right\}$ as

$$
\begin{aligned}
& r_{n}(z, \tau)=\frac{\rho_{n}(z)-\tau z^{(-1)^{n}} \rho_{n-1}(z)}{\pi_{n}(z)-\tau z^{(-1)^{n}} \pi_{n-1}(z)}, \\
& s_{n}(z, \tau)=\frac{\rho_{n}(z)-\tau \rho_{n-1}(z)}{\pi_{n}(z)-\tau \pi_{n-1}(z)} .
\end{aligned}
$$

We denote by $\overline{\Theta_{n}}(z)$ the closed disks determined by the quasi-approximants $r_{n}(z, \tau)$ and by $\overline{T_{n}}(z)$ the closed disks determined by the pseudo-approximants $s_{n}(z, \tau)$. The limiting disks are written as $\overline{\Theta_{\infty}}(z)$ and $\overline{T_{\infty}}(z)$, i.e.,

$$
\overline{\Theta_{\infty}}(z)=\bigcap_{n=1}^{\infty} \overline{\Theta_{n}}(z), \quad \overline{T_{\infty}}(z)=\bigcap_{n=1}^{\infty} \overline{T_{n}}(z) .
$$

We define for each $z$ outside $\hat{\mathbb{R}}$ the mapping $H_{z}: \mathbb{C} \rightarrow \mathbb{C}$ by

$$
w \longrightarrow w^{*}=H_{z}(w)=z^{-1}(1+w) .
$$

Theorem 5.1. For each $z \in \mathbb{C}-\mathbb{R}$, the mapping $H_{z}$ maps the disks determined by the even quasi-approximants with respect to $\nu$ onto the disks determined by the even pseudo-approximants with respect to $\mu$, i.e.,

$$
H_{z}\left(\overline{\Theta_{2 m}}(z)\right)=\overline{D_{2 m}}(z), \quad \text { for } m=1,2, \ldots
$$

Proof. Straightforward calculation, making use of (4.15)-(4.17), shows that

$$
\begin{aligned}
r_{2 m}(z, \tau) & =-1+\frac{z\left[\omega_{2 m}(z)-\tau z \omega_{2 m-1}(z)\right]}{\pi_{2 m}(z)-\tau z \pi_{2 m-1}(z)} \\
& =-1+z \frac{(1-\tau) \psi_{2 m}(z)+\left(g_{2 m}^{-1}+\tau g_{2 m}\right) \psi_{2 m-1}(z)}{(1-\tau) \varphi_{2 m}(z)+\left(g_{2 m}^{-1}+\tau g_{2 m}\right) \varphi_{2 m-1}(z)} \\
& =-1+z S_{2 m}\left(z, \frac{g_{2 m}^{-1}+\tau g_{2 m}}{\tau-1}\right) .
\end{aligned}
$$

The mapping $\tau \rightarrow\left(g_{2 m}^{-1}+\tau g_{2 m}\right) /(\tau-1)$ maps $\hat{\mathbb{R}}$ one-to-one onto $\hat{\mathbb{R}}$. Thus the boundary circle $\partial \Theta_{2 m}(z)$, obtained as the values of $r_{2 m}(z, \tau)$ for $\tau \in \hat{\mathbb{R}}$, equals the circle $\partial D_{2 m}(z)$, since $\partial D_{2 m}(z)$ is obtained as the values of $S_{2 m}(z, t)$ for $t \in \hat{\mathbb{R}}$. Consequently, $\partial D_{2 m}(z)$ equals $z^{-1}\left(1+\partial \Theta_{2 m}(z)\right)=H_{z}\left(\partial \Theta_{2 m}(z)\right)$. The mapping $H_{z}$ then obviously maps the interior of $\partial \Theta_{2 m}(z)$ onto the interior of $\partial D_{2 m}(z)$. This completes the proof.

Corollary 5.2. For each $z \in \mathbb{C}-\mathbb{R}$, we have

$$
H_{z}\left(\overline{\Theta_{\infty}}(z)\right)=\overline{D_{\infty}}(z) .
$$

Proof. This follows immediately from Theorem 5.1 and the definitions of $\overline{\Theta_{\infty}}(z)$ and $\overline{D_{\infty}}(z)$ when we take into account that the sequences $\overline{\Theta_{n}}(z)$ and $\overline{D_{n}}(z)$ are nested.

Theorem 5.3. For each $z \in \mathbb{C}-\mathbb{R}$, the mapping $H_{z}$ maps the disks determined by the odd pseudo-approximants with respect to $\nu$ onto the disks determined by the odd quasi-approximants with respect to $\mu$, i.e.,

$$
H_{z}\left(\overline{T_{2 m+1}}(z)\right)=\overline{\Delta_{2 m+1}}(z), \quad \text { for } m=0,1,2, \ldots
$$


Proof. We see from (2.12) that

$$
\varphi_{2 m+2}(z)-g_{2 m+2} \varphi_{2 m+1}(z)=h_{2 m+2} z \varphi_{2 m+1}(z)+f_{2 m+2} \varphi_{2 m}(z)
$$

and

$$
\varphi_{2 m}(z)+\frac{f_{2 m+1}}{g_{2 m+1}} \varphi_{2 m-1}(z)=\frac{1}{g_{2 m+1}} \varphi_{2 m+1}(z)-\frac{h_{2 m+1}}{z g_{2 m+1}} \varphi_{2 m}(z) .
$$

Substituting into the defining expressions for $\pi_{2 m+1}$ and $\pi_{2 m}$ and taking into account the relationships (2.14)-(2.16), we find

$$
\begin{aligned}
& \pi_{2 m+1}(z)-\tau \pi_{2 m}(z) \\
& \quad=g_{2 m+1}^{-1}\left(h_{2 m+2} g_{2 m+1}-\tau\right)\left[\varphi_{2 m+1}(z)-\frac{\left(\tau h_{2 m+1}-g_{2 m+2}\right)}{\left(h_{2 m+2} g_{2 m+1}-\tau\right)} \cdot \frac{1}{z} \varphi_{2 m}(z)\right] .
\end{aligned}
$$

Similarly

$$
\begin{aligned}
& \omega_{2 m+1}(z)-\tau \omega_{2 m}(z) \\
& \quad=g_{2 m+1}^{-1}\left(h_{2 m+2} g_{2 m+1}-\tau\right)\left[\psi_{2 m+1}(z)-\frac{\tau h_{2 m+1}-g_{2 m+2}}{\left(h_{2 m+2} g_{2 m+1}-\tau\right)} \cdot \frac{1}{z} \psi_{2 m}(z)\right] .
\end{aligned}
$$

By using (4.15)-(4.17), we see that

$$
\begin{aligned}
s_{2 m+1}(z, \tau) & =-1+\frac{z\left[\omega_{2 m+1}(z)-\tau \omega_{2 m}(z)\right]}{\pi_{2 m+1}(z)-\tau \pi_{2 m}(z)} \\
& =-1+z R_{2 m+1}\left(z, \frac{\tau h_{2 m+1}-g_{2 m+2}}{h_{2 m+2} g_{2 m+1}-\tau}\right) .
\end{aligned}
$$

Arguing as in the proof of Theorem 5.1, we conclude that $\partial \Delta_{2 m+1}(z)$ equals the image by $H_{z}$ of the boundary circle $\partial T_{2 m+1}(z)$, and hence that $\overline{\Delta_{2 m+1}}(z)=$ $H_{z}\left(\overline{T_{2 m+1}}(z)\right)$.

Corollary 5.4. For each $z \in \mathbb{C}-\mathbb{R}$, we have

$$
H_{z}\left(\overline{T_{\infty}}(z)\right)=\overline{\Delta_{\infty}}(z) \text {. }
$$

Proof. This follows from Theorem 5.3 in the same way as Corollary 5.2 follows from Theorem 5.1.

We formally establish an invariance property of the limiting disks $\overline{D_{\infty}}(z)$ analogous to that of the limiting disks $\overline{\Delta_{\infty}}(z)$.

Theorem 5.5. The limiting disk $\overline{D_{\infty}}(z)$ is either a single point for every $z$ outside $\hat{\mathbb{R}}$ or a proper disk for every $z$ outside $\hat{\mathbb{R}}$.

Proof. This follows immediately from Corollary 5.2 and the fact that the disks $\overline{\Theta_{\infty}}(z)$ have this invariance property.

The measure $\sigma$ given by

$$
d \sigma(t)=t^{2} d \mu(t)
$$

is a solution of a SSMP when $\mu$ is, and vice versa. We introduce the mapping $G_{z}$ of $\mathbb{C} \rightarrow \mathbb{C}$ as

$$
G_{z}(w)=H_{z}\left(H_{z}(w)\right)=\frac{1+z+w}{z^{2}} .
$$

The following result is an immediate consequence of Corollaries 5.2 and 5.4. 
Theorem 5.6. The mapping $G_{z}$ maps the limiting disks determined by the quasi-approximants with respect to $\sigma$ onto the limiting disks determined by the quasi-approximants with respect to $\mu$, and the limiting disks determined by the pseudo-approximants with respect to $\sigma$ onto the limiting disks determined by the pseudo-approximants with respect to $\mu$.

\section{Solutions of the SSMP}

We recall that $F(z, \mu)$ denotes the Stieltjes transform of $\mu$ at $z$ (see (1.5)). We also recall that the lens-shaped region $\overline{L_{\infty}}(z)$ is defined as the intersection $\overline{\Delta_{\infty}}(z) \cap \overline{D_{\infty}}(z)$ (see (3.13)).

Theorem 6.1. The values of the Stieltjes transform at $z$ of all the solutions of the SSMP exactly cover $\overline{L_{\infty}}(z)$, i.e.,

$$
\overline{L_{\infty}}(z)=\{w \in \mathbb{C}: w=F(z, \mu) \text { for some solution } \mu \text { of the SSMP }\} \text {. }
$$

Proof. We have already seen (in Section 3) that for every point $w$ in $\overline{L(z)}$, there is a solution $\mu$ of the SSMP associated with the sequence $\left\{c_{n}\right\}$ such that $w=F(z, \mu)$.

Let $\mu$ be an arbitrary solution of the SSMP associated with the sequence $\left\{c_{n}\right\}$. Then the measure $\nu$ defined by (4.2) is a solution of the SSMP associated with the sequence $\left\{\gamma_{n}\right\}$, and $a$ fortiori $\nu$ is a solution of the corresponding SHMP. Thus, $F(z, \nu) \in \overline{\Theta_{\infty}}(z)$. Direct calculation shows that

$$
F(z, \nu)=-1+z F(z, \mu)
$$

and, hence,

$$
F(z, \mu)=H_{z}(F(z, \nu)) \text {. }
$$

It follows from Corollary 5.2 that $F(z, \mu) \in \overline{D_{\infty}}(z)$. Since $\mu$ is a solution of the SHMP for the sequence $\left\{c_{n}\right\}$, we also have $F(z, \mu) \in \overline{\Delta_{\infty}}(z)$. Thus, $F(z, \mu) \in \overline{\Delta_{\infty}}(z) \cap$ $\overline{D_{\infty}}(z)=\overline{L_{\infty}}(z)$, which completes the proof.

Obviously, the SSMP is determinate if, and only if, $\overline{L_{\infty}}(z)$ reduces to a single point for $z$ outside $\hat{\mathbb{R}}$. We now give the following characterization.

Theorem 6.2. The SSMP is determinate if, and only if, at least one of the limiting disks $\overline{\Delta_{\infty}}(z), \overline{D_{\infty}}(z)$ reduces to a single point.

Proof. Obviously, the condition of the theorem is sufficient. Now, assume that the SSMP is determinate and that $\overline{\Delta_{\infty}}(z)$ is a proper disk. We need to show that $\overline{D_{\infty}}(z)$ reduces to a point. A proof of this is given in [31]. For completeness, we briefly sketch this central argument.

We set

$$
\varepsilon_{n}(z)=\frac{\psi_{n}(z) \varphi_{n-1}(z)-\psi_{n-1}(z) \varphi_{n}(z)}{\left(\bar{z} z^{-1}\right)^{(-1)^{n}} \varphi_{n}(z) \overline{\varphi_{n-1}(z)}-\varphi_{n-1}(z) \overline{\varphi_{n}(z)}}
$$

and

$$
e_{n}(z)=\frac{\psi_{n}(z) \varphi_{n-1}(z)-\psi_{n-1}(z) \varphi_{n}(z)}{\varphi_{n}(z) \overline{\varphi_{n-1}(z)}-\varphi_{n-1}(z) \overline{\varphi_{n}(z)}}
$$


It follows by elementary properties of Möbius transformations that the radius of $\overline{\Delta_{n}}(z)$ equals $\left|\varepsilon_{n}(z)\right|$, and the radius of $\overline{D_{n}}(z)$ equals $\left|e_{n}(z)\right|$. We find that

$$
\frac{1}{e_{n}(z)}-\frac{1}{\varepsilon_{n}(z)}=\frac{\left[1-\left(\bar{z} z^{-1}\right)^{(-1)^{n}} \frac{\overline{\varphi_{n-1}(z)}}{\varphi_{n-1}(z)}\right]}{\left[\frac{\psi_{n}(z)}{\varphi_{n}(z)}-\frac{\psi_{n-1}(z)}{\varphi_{n-1}(z)}\right]} .
$$

We have assumed that $\overline{\Delta_{\infty}}(z)$ is a proper disk. Then the radius of $\overline{\Delta_{n}}(z)$ tend to a positive value, and hence $1 / \varepsilon_{n}(z)$ is bounded. We also have assumed that $\overline{L_{\infty}}(z)$ reduces to a single point. Since $\psi_{2 m}(z) / \varphi_{2 m}(z)=R_{2 m}(z, 0)=S_{2 m}(z, 0)$ and $\psi_{2 m+1}(z) / \varphi_{2 m+1}(z)=R_{2 m+1}(z, 0)=S_{2 m+1}(z, 0)$, in general, converge to the two common end points of the $\operatorname{arcs} \Omega_{\infty}(z)$ and $\Gamma_{\infty}(z)$, it follows that in our situation the sequence $\left\{\psi_{n}(z) / \varphi_{n}(z)\right\}$ converges. (This convergence is a well-known property of the even and odd approximants $\psi_{n}(z) / \varphi_{n}(z)$ associated with a determinate SSMP; see $[6,21,27-28]$.) Consequently, the right-hand side of $(6.6)$ is unbounded (for every $z$ outside $\hat{\mathbb{R}}$ ). It follows that $1 / e_{n}(z)$ is unbounded, which means that the monotonic sequence $\left|e_{n}(z)\right|$ tends to zero. Hence, $\overline{D_{\infty}}(z)$ reduces to a single point.

This completes the proof.

Theorem 6.3. The SSMP associated with $\left\{c_{n}\right\}$ is determinate if, and only if, at least one of the two SHMPs associated with $\left\{c_{n}\right\}$ and $\left\{\gamma_{n}\right\}$ is determinate.

Proof. The statement is equivalent to the statement that at least one of the disks $\overline{\Delta_{\infty}}(z)$ and $\overline{\Theta_{\infty}}(z)$ reduces to a point, for some $z$ or equivalently for every $z$ outside $\hat{\mathbb{R}}$. By Corollary $5.2, \overline{\Theta_{\infty}}(z)$ reduces to a point if, and only if, $\overline{D_{\infty}}(z)$ reduces to a point. The result now follows from Theorem 6.2 .

\section{References}

1. N. I. Akhiezer, The Classical Moment Problem and Some Related Questions in Analysis, Hafner, New York, 1965.

2. C. Berg, Markov's theorem revisited, J. Approx. Theory 78 (1994), 260-275.

3. $\_$Indeterminate moment problems and the theory of entire functions, J. Comp. Appl. Math. 65 (1995), 27-55.

4. C. Berg and H. L. Pedersen, On the order and type of the entire functions associated with an indeterminate Hamburger moment problem, Ark. Mat. 32 (1994) 1-11.

5. C. Berg and G. Valent, The Nevanlinna parametrization for some indeterminate Stieltjes moment problems associated with birth and death processes, Methods Appl. Anal. 1 (1994), 169-209.

6. C. M. Bonan-Hamada, W. B. Jones, O. Njåstad, and W. J. Thron, A class of indeterminate strong Stieltjes moment problems with discrete distributions. In: Orthogonal Functions, Moment Theory, and Continued Fractions: Theory and Applications, (W. B. Jones and A. Sri Ranga, eds.), Marcel Dekker, New York, 1998, pp.31-55.

7. A. Bultheel, P. Gonzalez-Vera, E. Hendriksen, and O. Njåstad, Orthogonal Rational Functions, Cambridge University Press, 1999.

8. T. S. Chihara, On indeterminate Hamburger moment problems, Pacific J. Math. 27 (1968), 475-484.

9. __ An Introduction to Orthogonal Polynomials, Gordon and Breach, New York, 1978.

10. _ Indeterminate symmetric moment problems, J. Comp. Appl. Math. 85 (1982), 331-346.

11. L. Cochran and S. Clement Cooper, Orthogonal Laurent polynomials on the real line. In: Continued Fractions and Orthogonal Functions, (S. Clement Cooper and W. J. Thron, eds.), Marcel Dekker, New York, 1994, pp. 47-100. 
12. V. A. Fil'stinskii, The power moment problem on the entire axis with a finite number of empty intervals in the spectrum, Zap. Mekh. -Mat. Fak. Khar'kov. Gos. Univ. Khar'kov Mat. Obshch. 30 Ser. 4 (1964), 186-200 (in Russian).

13. G. Freud, Orthogonal Polynomials, Pergamon Press, New York, 1971.

14. A. A. Gonchar, On the convergence of generalized Padé approximants of meromorphic functions, Mat. Sb. 98 (1975), 564-577; English trans.: Math. USSR - Sb. 27 (1975), 503-514.

15. H. Hamburger, Über eine Erweiterung des Stieltjesschen momentproblems, Parts I, II, III, Math. Annalen 81 (1920), 235-319; 82 (1921), 120-164; 82 (1921), 168-187.

16. W. B. Jones, O. Njåstad, and W. J. Thron, Two-point Padé expansions for a family of analytic functions, J. Comp. Appl. Math. 9 (1983), 105-123.

17. — Continued fractions and strong Hamburger moment problems, Proc. Lond. Math. Soc. 47 (1983), 363-384.

18. _ Orthogonal Laurent polynomials and the strong Hamburger moment problem, J. Math. Anal. Appl. 98 (1984), 528-554.

19. W. B. Jones and W. J. Thron, Orthogonal Laurent polynomials and Gaussian quadrature. In: Quantum Mechanics in Mathematics, Chemistry and Physics, (K. E. Gustafson and W. P. Reinhardt, eds.), Plenum Publishing Company, 1980, pp. 449-445.

20. __ Survey of continued fraction methods of solving moment problems and related topics. In: Analytic Theory of Continued Fractions, (W. B. Jones, W. J. Thron, and H. Waadeland, eds.), Lecture Notes in Mathematics 932, Springer-Verlag, Berlin, 1982, pp. 4-37.

21. W. B. Jones, W. J. Thron, and H. Waadeland, A strong Stieltjes moment problem, Trans. Amer. Math. Soc. 261 (1980), 503-528.

22. H. J. Landau (ed.), Moments in Mathematics, Proc. Symposium Appl. Math. 37, Amer. Math. Soc., Providence, RI, 1987.

23. G. Lopes [G. Lopez Lagomasino], Conditions for convergence of multipoint Padé approximants for functions of Stieltjes type, Mat. Sb. 98 (1978), 69-83; English trans.: Math. USSR-Sb 35 (1979), 363-376.

24. G. Lopez Lagomasino and A. M. Finkelshtein, Rate of convergence of two-point Padé approximants and logarithmic asymptotics of Laurent-type orthogonal polynomials, Constr. Approx. 11 (1995), 255-286.

25. R. Nevanlinna, Asymptotische Entwicklungen, Beschränkte Funktionen und das Stieltjessche Momentproblem, Ann. Acad. Sci. Fenn. A 18 (1922).

26. — Über beschränkte analytische Funktionen, Ann. Acad. Sci. Fenn. A 32 (1929), 1-75.

27. O. Njåstad, Solutions of the strong Stieltjes moment problem, Methods Appl. Anal. 2 (1995), 320-347.

28. —_ Extremal solutions of the strong Stieltjes moment problem, J. Comp. Appl. Math. 65 (1995), 309-318.

29. - Solutions of the strong Hamburger moment problem, J. Math. Anal. Appl. 197 (1996), $227-248$.

30. —_ Remarks on canonical solutions of strong moment problems. In: Orthogonal Functions, Moment Theory, and Continued Fractions: Theory and Applications, (W. B. Jones and A. Sri Ranga, eds.), Marcel Dekker, New York, 1998, pp. 359-374.

31. _ - Strong Stieltjes moment problems, In: Continued Fractions: From Analytic Number Theory to Constructive Approximation, (B. Berndt and F. Gesztesy, eds.), Contemporary Mathematics Series, American Mathematical Society, Providence, R.I., 1999.

32. O. Njåstad and $\mathrm{W}$. J. Thron, Unique solvability of the strong Hamburger moment problem, J. Austral. Math. Soc. Series A 40 (1986), 5-19.

33. H. L. Pedersen, The Nevanlinna matrix of entire functions associated with a shifted indeterminate Hamburger moment problem, Math. Scand. 74 (1994), 152-160.

34. _ Stieltjes moment problems and the Friedrichs extension of a positive definite operator, J. Approx. Theory 83 (1995), 289-307.

35. __ La paramétrisation de Nevanlinna et le problème des moments de Stieltjes indeterminé, Expo. Math. 15 (1997), 273-278.

36. A. Sri Ranga, $\hat{J}$-fractions and strong moment problems. In: Analytic Theory of Continued Fractions II, (W. J. Thron, ed.), Lecture Notes in Mathematics 1199, Springer-Verlag, Berlin, 1986, pp.269-284. 
37. M. Riesz, Sur le problème des moments, I, II, III, Arkiv för Matematik, Astronomi och Fysik 16 (1922); 16 (1922); 17 (1923).

38. J. A. Shohat and J. D. Tamarkin, The Problem of Moments, Mathematical Surveys 1, Amer. Math. Soc., Providence, R.I., 1943.

39. T. J. Stieltjes, Recherches sur les fractions continues, Ann. Fac. Sci. Toulouse 8 (1894), 1-122; 9 (1894), 1-47; Oeuveres 2, 402-566.

40. M. H. Stone, Linear Transformations on Hilbert Space and Their Applications to Analysis, Amer. Math. Soc. Publ. 15, New York, 1932.

41. K. I. Švecov, On Hamburger's moment problem with the supplementary requirement that masses are absent on a given interval, Commun. Soc. Math. Kharkov 16 (1939), 121-128 (in Russian).

42. W. Van Assche, The impact of Stieltjes' work on continued fractions and orthogonal polynomials. In: T. J. Stieltjes, Collected Papers, Springer-Verlag, Berlin, 1993.

Department of Mathematical Sciences, Norwegian University of Science and Technology, N-7491 TRONDHEIM, NORWAY 